\title{
Precision Medicine: Neuropsychodynamic Psychiatry Model of Major Depressive Disorder
}

\author{
Huang Yaqi \\ Department of Applied Mathematics, The Hong Kong Polytechnic University, Hong Kong \\ ${ }^{*}$ Corresponding author. Email: 16095452d@connect.polyu.edu
}

\begin{abstract}
Major Depressive Disorder (MDD) is one of the most extensive types of psychiatric disorders. It refers to various symptoms and considerable rates of relapse, where episodes rarely resolve completely even with intensive psychiatric care. There has been growing concern over the ineffectiveness in treating MDD in reductionist domination in the psychiatry domain. The recent development of neuropsychodynamics offers an integrative model incorporating a holistic understanding of the human mind in terms of neuroscience and psychoanalytic theories and provides a potentially effective cure for MDD. In this review, MDD in the neuropsychodynamic model is manifested as an abnormal operation of the self. The chief feature refers to the increasing inward focus of the self and decreasing outward direction of the environment. To argue for the necessity of utilizing neuropsychodynamic methodology in assessing and treating MDD, the advantages of the model were discussed.
\end{abstract}

Keywords: Neuropsychodynamic, Precision Medicine, Psychiatry Model, Major Depressive Disorder

\section{INTRODUCTION}

Major Depressive Disorder (MDD) is one of the most common psychiatric diseases, contributing to the global burden of disease [1], affecting more than 264 million people [2]. It contributes to approximately 800,000 suicide deaths per year [1]. MDD has a high prevalence and affects $20.6 \%$ of individuals in a lifetime course [3]. Mainstream psychiatry manual states that an individual who shows enough symptoms for 2 weeks, including depressed mood, anhedonia, weight loss, insomnia or hypersomnia, loss of energy, and indecisiveness, is considered for MDD [4].

The risks of MDD are polygenic and multifold. MDD has heritability that ranges from $8.75-40 \%$ [5], imposing a threefold risk for first-degree relatives of MDD individuals [6]. Although the risks involve various genes with minor effects [7], recent studies have confirmed 178 genomic risk loci for MDD [8]. Moreover, environmental correlates such as stressful life events and low social support are also significant contributors [9]. Furthermore, other areas such as neuroendocrinology [10], inflammation levels [11], and functional brain circuits [12] also have outstanding correlates.

Common treatments for MDD include psychotherapy and antidepressants (used alone or in combination), depending on the severity of symptoms [4] determined by trial and error [13]. Notwithstanding the tremendous effort in investigating MDD, current clinical practice promises far from optimal efficacy. As one of the most commonly misdiagnosed mental disorders [14], up to $50-60 \%$ of patients with MDD do not receive adequate treatment [15], while others receive poorly designed personalized treatment for comorbid issues [16]. However, antidepressants, if correctly prescribed, merely demonstrate moderate efficacy [17] with nonegelible adverse effects and delayed response [18], leaving $20-30 \%$ of patients (TRD) resistant to treatment [19].

This inefficiency may be (at least in part) attributed to the puzzling multifactorial etiology and phenotype heterogeneity of MDD. One aspect that hinders researchers from obtaining an integrated perspective on MDD may be the reductionism that dominates research directions and funding policies in clinical psychology and psychiatry [20]. The unbalanced analytic approach in the present neuroscience context [21] or a single target-focused procedure is certainly unsuited for the polygenic nature of neuro-psychic dynamics that drives MDD; neither descriptive psychology alone can explain the causal relationships between intertwined factors. To fully capture the complexity of neuro-psychic ailments 
such as MDD, it is of significant necessity to see the "real patterns" of people [22] and combine biological and psychological understanding to obtain a "holistic approach" [22] or the so-called neuropsychodynamic approach.

The term "neuropsychodynamics" or "psychodynamic psychiatry" [23] is an approach steeped into psychoanalytic theories founded by Sigmund Freud, the models majorly investigating unconsciousness of buried feelings, memories, dreams, and fantasies driving conflicts between wishes and anxieties [24]. As a school of psychoanalysis, psychodynamics is considered a broader domain for psychotherapy procedures offering less intensive and more supportive-interpretive treatment than psychoanalysis psychotherapy [23] that has been developed for treating a wide range of disorders [24]. While the efficacy of psychodynamic therapy (PDT) is debatable, [25] demonstrates the noninferiority of PDT in treating MDD among other psychotherapies. Intensive short-term PDT (ISPDT) has shown significant [26] and sustained [27] improvement in MDD; long-term (LTDP) is superior to other therapies in treating chronic depression in terms of personality and social functioning [28].

PDT places paramount value on the individuals' inner world. PDT therapists emphasize transference and countertransference, resistance to therapy, functions and unconscious forces behind the symptoms and behaviors [29]. Moreover, PDT-oriented treatment considers specific characteristics of the employment of developmental perspective and person-centered proposition [30]. Despite several PDT theories of MDD mental representations, it remains divorced and thus limited in unraveling the mystery of the human brain and its complications; this constraint also applies to the dominance of descriptive or biology explanations. An integrative approach, namely neuropsychodynamics, should replace such reductionist methods involving underlying neural mechanisms for intrapsychic structures and internal object relations [31]. In this context, neuroscience may offer biological justifications to psychodynamic notions, whereas PDT theories may offer a crucial conceptual structure for neurological discoveries [32].

\section{THE UNIQUENESS AND ADVANTAGES OF NEUROPSYCHODYNAMICS}

A well-designed individual psychiatric treatment demands the clinical practitioner to comprehend that a human being is a feeling organism, and the objective and subjective experience should be considered with equal weight. By aligning neuroscience and psychoanalysis theories, neuropsychodynamics possesses virtues from both sides, which form its uniqueness.
Neuropsychodynamics shares one of Freud's cornerstones of thinking: psychic determinism. The definition of this term has extended from its original assertation from psychodynamics, that apart from the theory of unconscious forces writing our life scripts, psychiatric symptoms and behaviors also illustrate (but not limit) our adaptations to specific demands of our unconsciousness that blends our early attachment, complexes, object relations, defense mechanisms, fixations, and cathexis throughout the development of the self with aspects of genes, biological, and neurological factors [23]. Neuropsychodynamics emphasizes this causality of human emotions and actions that these complex and multiple causes need careful examination before claiming that symptoms have no meaning other than themselves.

In this sense, neuropsychodynamics faces the brainmind problem with the issue of the construct of "person", in which the understanding of the "person" undoubtedly confronts uncomplicated classification. According to Gabbard [31], the principle of determinants of a person in the neuropsychodynamic method should involve the subjective experience, a coterie of inner conflicts and internalized interactions, physical attributes, "brain as a product of genes", neural system by accumulative life incidents, cultural milieu, social and economic status, religion, and cognitive style.

Of paramount significance, the subjective experience refers to "a unique historical narrative that is filtered through specific meanings" [23] that could offer invaluable evidence in the clinical setting. In contrast to descriptive psychiatry, neuropsychodynamics does not regard symptoms as guidelines but rather the final result of the mechanism that protects and reflects the inner world of an individual [33]. Practitioners need to determine the starting point and various developmental trajectories for a complete understanding of the person. In a metaphorical way to depict the limit of such descriptive methods, one could argue that symptoms are merely projections that suggest nothing but the shape of an object under a beam of light; other facets, namely, the volume, structure, and object depth hiding in the back of the façade remain unknown. Accordingly, the reductionist philosophy again, solely examining the defining features of patients and analyzing them by statistical methods (finding correlations of external manifestation), will inevitably fail to perceive the overall reality that psychiatric practice strives to know.

In neuropsychodynamics, considering life history also means evaluating patient self-disclosure from a cognitive perspective. As cognitive science grasps, the human psyche is a continuing state of change that entails dynamic interaction with the environment with relabeling and re-interpreting personal experiences [34]. Present-day clinical reports (similar to any self-report at any moment) are consequences of unconscious forces 
that come from memories of earlier situations and need conditional consideration subject to a specific time, space, and developmental course. Therefore, under the neuropsychodynamic procedure, convoluted constructions behind the patient claims will be recognized and assessed with care and appropriate skepticism, and hopefully, further inquiries will lead to a more insightful diagnosis.

Moreover, the developmental perspective in neuropsychodynamics captures the wisdom from the epigenetic theory and psychodynamic bibliography studies. On the one hand, moving beyond the naturenurture debate, a consensus has been reached that genes are inherited and show constant interplay with the environment. Although specific candidate genes are responsible for psychiatric disorders such as autism spectrum disorder and schizophrenia [35], other psychological disturbances (e.g., MDD) have their fundamental roots in neuroplasticity [36]. Apart from neurological studies, integral physical health such as immune system functioning is also considered in neuropsychodynamics. For recent evidence, Borsini et al. [11] stated that increased production of inflammatory cytokines directly contributes to depressive symptoms.

On the other hand, personal bibliography or individual life experiences, namely, childhood events, trauma, patterns of interaction within the family, and other related events, are of considerable etiological importance contributing to the development of the self. These incidents are crucial in assessing and interpreting the cause of psychiatric symptoms [35]. In addition to experiences after birth, a recent study confirmed that trauma exposure during pregnancy could affect the offspring, leading to social deficits and depressive behaviors [38]. In this proposition, temperament theory and attachment theory are outstandingly significant subgroups. Humans are born with the initial temperament that is chiefly inherited and yet affected by parental styles [39]. Temperament types impact how parents treat infants in a "goodness-of-fit" fashion [40], thus affecting the mother-child relationship quality. Inherited temperament and childhood experiences are strong predictors of adulthood personality and psychiatric behaviors with other events and potential trauma [41]. Later in childhood, attachment is created between the child and the caregiver. Attachment schemes are acquired during early childhood and stay relatively steady throughout the lifetime, affecting neural mechanisms as consequences [42]. Exhaustive understanding of the above historical elements of patients serves great importance under clinical practice scenarios that aim to achieve a precise diagnosis, pathological analysis, and individualized treatment plan.

Lastly, a unique feature that distinguishes neuropsychodynamics from other conventional psychiatry approaches is the intensive utilization of transference and countertransference as practical diagnosis tools. However, this term is not novel in the domain of psychodynamics. When transference (countertransference) occurs, the patient (the therapist) experiences earlier psychological episodes or past relationships with the therapist (the patient) as of the current relationship [43]. The value of such a process lies in the fact that even consciousness is not always declarative, not to mention the unconscious stories [44]. Through (counter) transference, unconscious processes automatically occur, unraveling internal representations of current objects (the therapeutic setting, relationship, characteristics of the other person) inaccessible by conscious self-report or diagnosis via descriptive manner. Being aware of patient transference and countertransference assists the physician in improving the diagnostic process or implies richer evidence by subsequent analysis [45].

\section{NEUROPSYCHODYNAMIC MODEL OF DEPRESSION}

The depression model of the neuropsychodynamics discipline comes from Freud's theory of Melancholia that stresses ego-regression from object cathexis to narcissism and indicates the depletion of associated reality basis. Freud [46] explained that "the objectrelationship was shattered" in depression, which did not result in "the normal one of a withdrawal of the libido" and displacement with a new one, but a state that the libido "was withdrawn into the ego." Thus, the ego "established an identification" with "the abandoned object," and the object loss "was transformed into an ego-loss." Melancholia (depression) is different from mourning (normal mourning process) in that it is comparable to an absence of necessary mourning. More specifically, when facing object loss, if the individual does not regard "the world itself is poor and empty," the self-regard is still processed in healthy object relations with his ego guarded. The person becomes self-absorbed and impoverishes himself with a "diminution of his ego." He "reproaches himself" and represents himself as "worthless, incapable of any achievement and morally despicable" [46]

From a functional perspective, Freud's proposition is consistent with the present studies [47] on depression, which show that depressive symptoms are useful reactions to bereavement that could protect the ego. However, this process would develop into other dysfunctional effects, e.g., an absence in emotional reflection, a dominance of pessimistic and irrational cognitions, and extreme withdrawal behaviors, such as social withdrawal and diminished ability to make decisions. The relation between object loss and the loss of the self is of outstanding importance in the evolution of depression. The corresponding neurological aspects 
contribute to validating the underlying psychoanalytic and psychodynamic forces to interpret depression.

The neuropsychodynamic model of MDD is a mental-oriented particulars of localization, which considers the biological, psychological, and social dimensions of MDD [48]. Though studies and theories offer various explanations on this topic, this paper aims to summarize the depression trajectory through three psychodynamic stages, namely, early object loss, introjection of the loss object, and loss of actual object relations [47].

Phase 1: Early Object Loss. As argued earlier, depression occurs due to the event of loss of an object. This "early" loss could be event-specific. However, it generally refers to early object loss during childhood, using a metaphorical approach [49]. In broader psychology theories, early loss in childhood serves as a multidimensional susceptibility of individuals to progress MDD in life afterwards [50]. During the critical period of synapses growth, the early loss will cause fixation on the mental characterization of lost objects, forming an "open wound" [46] and taking libido into the self when reactivated by later loss events.

This proposition for reactivation of early trauma is hypothesized to correlate with increased resting-state activity in the thalamus [51], amygdala [52], medial temporal lobe and posterior cingulate cortex [53], lateral anterior cortical midline regions [54], and subcortical and cortical midline regions [55]. A recent study demonstrates that this hyperactivity in different areas could signal distinct depression biotypes [56]. In psychodynamic terms, this resting state activity dysbalance correlates to the tendency of psychological compensation, characterized by an increase of selfreferential processing associated with negative affective valence resulting from object loss or disappointment in childhood [48]. Such traumatic events include incidents of actual loss, parents' separation, and abuse in any means (corresponding to the loss of primary narcissism), exhibiting greater vulnerability for later MDD onset [57].

Following object loss, the child would attempt desperately to relate the self to the lost object to sustain a self-object relationship [48]. If the trauma persists, the child will eventually experience object loss as a selfobject due to the absence of that actual object that is supposed to serve as the retention of free libido [58]. Simultaneously, a possible hyperactivation may be induced in the regions mentioned earlier, finally contributing to an imbalance in other consecutive areas with hypoactivation. To specify, major hyperactivation occurs in the anterior midline regions primarily involved in the processing of self-reference of different stimuli, resulting in hypoactivation in the posterior midline regions associated with the operation of non-self-related stimuli [59]. Accompanied by such early object loss, it would interfere with the development of the essential parts of the default mode network (DMN) when aiming to produce an object cathexis, adding risks for later depression [60].

Phase 2: Introjection of Object Loss. Following the increased resting-state activity in the cortical midline regions, a reduced resting-state stimulus interaction of the current object and growing autobiographic memory retrieval of the past object occurs [48]. This course includes an interaction between intrinsic brain activity and stimulus-induced (inside and outside) activity. Reduction in this process will produce diminished selfreferential processing and affective attribution of current stimuli, thereby reducing reference to the current object of a patient.

The growing reactivation of autobiographic recollections of the early objects refers to higher selfreferential processing and affective attribution of past stimuli, i.e., the patient will experience increased reference to the past object. This increased inner focus contributes to the inability of the depressive patient to focus on the outer world; they no longer subjectively perceive the actual stimuli, manifesting "feeling of feelings" where "feelings" involve earlier experienced stimuli. More specifically, patient's attentiveness transfers from the exteroceptive sensory system to the interoceptive sensory system that mainly processes somatic stimuli [48]. The actual stimuli can no longer relate to the patient's self and attach with emotional valence appropriately. As a result, the psychic energy used to produce self-reference and the link with effects is related to earlier stimuli, i.e., object loss in the past. In psychopathology, this course suggests that a subjective experience of the past prevails over the future, and the patient usually reports feelings of numbness, increased self-focus, and anhedonia for current objects [61].

Arguably, concentrating on past object loss while neglecting current existing objects indicates that the depressive patient encounters increased introjection, coupled with negative emotions [62]. In psychodynamic terms, introjection is one type of internalization [63], a process or defense mechanism that depressive patients majorly employ [64]. Like other defense mechanisms, it shows an unconscious adaptation to protect the mind from danger within and without [65]. Introjection is identified by the alteration of object focus from the outside to the inside in subjective experience [66] that entails taking a value or regulatory process but not accepting it as own, resulting in internal control regulation instead of self-regulation [63]. Regarding object relations, depressive patients' relation orientation is no longer directed to the outside but the inside. They tend to internalize their conflicts and shift the aggression against themselves instead of running towards others [67]. It may be hypothesized that these disturbances in 
self-reference are associated with abnormal reciprocal modulation during emotional-cognitive interaction [47].

Accompanied with introjection, at this stage, patients will develop other salient depressive symptoms, e.g., rumination and negative bias, since agency and synthesis within oneself are harmed, affecting different themes such as self-protection, self-assertion, and selfexpansion [64]. To validate in neurology terms, such phenomenon is partially due to alterations in DMN functioning. This increased functional connectivity in the cortical parts of DMN is highly correlated to dense rumination, where depressive patients experience repetitive stressful styles of thinking about unfinished concerns [68]. Reduced resting-state connectivity in this phase causes deficits in the patient's reward system, with significantly reduced activation for wins compared with a loss in regions such as bilateral ventral [69]. It also impairs decision-making in rewarding conditions [70]. Since DMN serves as the neurological basis for the self, such dysbalance impairs the patient's self-related processing such as reflection, emotion, and selfreference [71]. Negative self-regard bias emerges due to deterioration of the cognitive ability by the stated disruption in DMN functional connectivity [72].

Phase 3: Loss of Actual Object Relations. Due to reduced stimulus-rest interaction of the current object, depressive individuals have difficulties connecting exteroceptive and interoceptive stimuli [73], orienting cognitive targets outward [74], and modifying restingstate brain activity by interoceptive stimuli [75]. Consistently, loss of object relations and further reduced outside focus is derived. MDD patients may subsequently experience increased body focus of interoceptive origin that induces somatic-vegetative symptoms [76] and growing cognitive stress with selfobjects [77].

As the third psychodynamic central hallmark of depression, patient-object relationships are substituted using a regressive cathexis of the self, contributing to increased splitting in the self, and actual object relation experience becomes increasingly unimportant [47]. Freud's metaphor [46] explains this stage as "constitute compensatory objects." Patients compensate for the loss of external self-objects by internal self-objects, implying that the self is disconnected from the experienced importance of actual object relation eventually. Depressed individuals suffer from markedly biased harmful cognitive patterns and ruminations, experienced as tormenting and resulting from the extending cognitive processing of the self with ruminations and a dysbalance between the experiential and the analytical self-focus [75].

The above psychodynamic features at this phase may associate with neurological foundations. Splitting in the self may relate to diminished ability to perform cognitive tasks that require the brain to respond to exteroceptive stimuli as the self is functioning in a self-absorbing pattern that merely interacts with the outside effectively. This finding could be expressed by increased activation in the dIPFC (more significant efforts) during working memory and cognitive processing tasks in depressed people compared to healthy people. In contrast, the vmPFC of the depressed is hyperactive at rest (in healthy individuals, this duo should express the opposite) [78]. This imbalance indicates inefficiency in the depressed's cognitive functioning and abnormally intensive processing of emotions and sensory, social, and selfrelated information [79] corresponding to the pathological analytical self-focus previously mentioned. This impairment could also be explained by limited taskevoked connectivity between the ACC (serves attentional orientating and affective processing) and task-negative networks, marked by inflexibility in local efficiency of a key ACC node in CEN [80], which is compatible with the depressed's stiffness in shifting cognitive control joining internal and external stimuli. The self can no longer be "filled" with emotions and cognitions derived from the outside but remains "empty" and emotionally inhibited, evolving into ego-emptiness and ego-inhibited, respectively [47].

\section{NEUROPSYCHODYNAMIC TREATMENT ESSENTIALS}

The neuropsychodynamic model strongly recommends individualized procedures. It is widely acknowledged that MDD shares multidimensionality and heterogeneity, proving the crucial significance of adapting appropriate neurological and psychotherapeutic treatments to the specific condition in each case [82]. Neuropsychodynamics addresses the idea of MDD as psychosomatics of emotion modulation. The primary aim of therapy is to overcome immature defense mechanisms that pull the patient's cognition into vicious cycles and finally damage the interpersonal and psychosomatic body [47]. However, though the therapeutic goal mostly aligns with PDP [82], neuropsychodynamics calls for more complex and nuanced interventions.

To put it more practically, while examining cognitive patterns in MDD, the therapist shall not solely maintain psychodynamic explanations that the patient exhibits delusional, illogical beliefs requiring empathy and emotional support, which often fail to offer specific instructions to tackle depressive thinking actively. Consistently, empirical evidence on the efficacy of PDP mainly exists in the long-term scale [83] where variables other than PDP such as positive life events, new intimate relationships, and escape from a toxic working environment would emerge and impact the patient. This heterogeneity and uncertainty of grey data would result in statistical inaccuracy. To make amends in the current 
SPDP practice, the neuropsychodynamic therapist should be aware of factors such as cognitive symptoms that may relate to various neurological functions causing several consecutive changes in feedback processing, where appropriate medication and an adjustment in daily routines (e.g., diet plan, exercise intensity, CBT guide) are in priority. Thus, the therapist may need to take active steps to "attune" patient neuron connections instead of simply listening to those who suffer.

Such concerns appear similarly in the other polarity. For psychiatrists who mainly examine the mind using a reductionist approach, one should be aware that medication may alleviate specific pain symptoms. However, it may not promise healthier cognition or the employment of mature defense mechanisms (where problems can only be transcended by the patient's maturing personality construct) that free MDD symptoms and protect the patient from future relapses. Disregarding the patient's inner-psychic world would render psychiatrists in clinical practice without sufficient empathy and acquisition of the broader context of MDD. Physicians should fully capture the mechanism behind the "voluntary" or top-down modulation and recognize the "involuntary," pathological neurology foundation of such negative bias with roots in the patient's personality and personal history, offering constructive suggestions with empathy. A psychodynamic mindset is also helpful in exploring and assessing a patient's whole-person information that psychiatrists have at best acquired.

Precise assessment and refined interventions, if provided, are not the destination of a well-conducted neuropsychodynamic therapy. It is important to note that the neuropsychodynamics model defies formulaic and mechanic diagnosis subject to rigid, outdated, and stigmatized standards. Operations and formulation of the treatment plan should incorporate basic empathy, interdisciplinary knowledge of patient information, including but not limited to pathological particulars, personal history, and cultural background. The ability to appreciate the patient's freedom of choice, psychological or physiological requirements, and desire for short-term therapeutic goals consistent with values and realistic affairs is a quality needed in practitioners. The synergistic effects of pharmacological (top-down mechanisms) and psychotherapeutic (bottom-up agents) intervention also need consideration when applying combination therapies.

\section{DISCUSSION}

This paper presents a brief literature review of contemporary MDD and addresses research gaps in reductionist clinical ineffectiveness and insufficiency in a comprehensive recognition of MDD. Accordingly, the study discusses the virtues of neuropsychodynamics and elaborates a unique demonstration of MDD. The neuropsychodynamic model of MDD combines psychodynamic (psychoanalytic) theories, neurological concepts, and subject experience with developmental views. In this approach, MDD is depicted by the construct of the self and abnormal changes in selfexperience, where the loss of self in MDD results from introjecting earlier object loss to the self. Empirical neuronal mechanisms have supported this psychological alteration, leading to the development of neuropsychodynamic hypotheses of MDD. Specifically, early object loss (trauma) is related to increased restingstate activity, increasing susceptibility to reactivation of early loss memories. Such increasing activity further renders decreased resting-state stimulus interaction enhancing the introjection procedure, encumbering the self from interacting with exteroceptive stimuli. This "alienation" of the self and the environment leads to depressive symptoms, namely negative emotions, distorted cognitions, and habits of rumination. Apart from the abnormalities mentioned above, various other neurological pathologies are related to this MDD model. Significant problems exist in the dysbalance between the DMN system (the self) and PFC functioning (the self interacts with other objects), where reciprocal interaction between the two alters in the opposite direction compared to healthy brains, resulting in an unusual increment in internal mental contents and decreased actions aligned with the external environment. The intensified inward focus leads to intense ruminations, and the minor outward focus gives rise to distresses differentiating the self and the external stimuli, manifesting key symptoms of MDD.

Scholars have proposed integrating multiple psychiatry domains for many years [84]. However, it is practiced with inefficiency in the clinical environment due to enduring confusion of "languages" [85]. Psychodynamics, neurobiology, biological psychiatry claim different models of MDD, and all these disciplines apply distinctive concepts that seem incompatible with each other. Boeker, Hartwich, and Northoff [33] addressed a potential framework that incorporates commonly shared features necessary to bridge different approaches to overcome the burden. In addition to what Northoff [86] elaborated in this framework, expansion on the domain may better assist future studies in precision medicine consisting of spatiotemporal features. These characteristics may enable joint descriptions of psychodynamics (e.g., unconscious conflicts), psychopathology (e.g., cognitive impairment), neuropsychology (e.g., decreased frontoparietal connectivity), epigenetics (glucocorticoid receptor resistance), and overall physiological health (increased serum cytokine levels) found on the spontaneous functioning of the spatiotemporal model.

In the real-life implementation of neuropsychodynamics in treating MDD, other critical points need to be addressed. Concerning epistemology, 
risks would rise due to categorical errors in psychodynamic abstractions measured by neurological means. Therefore, the chief concern would be to term, classify, and "translate" concepts among different disciplines without reducing meaning. More significant problems would emerge with the transformation of subject experience and other individual contents into data. This term in mainstream science refers to generalizability, employment of technical measuring, and linkage to public procedure [87]. One possible solution would be revealing the same content and comparing individual experiences labeled via psychodynamic processes for similarities and differences across individuals. Thus, it is possible to validate and objectify them structurally [88] to perceive them as data in the scientific sense.

However, this model comprises unsolved limitations due to various constraints and shortcomings in the selected studies. The hardship of incorporating different "languages," as mentioned, inevitably results in skepticism in measuring unconscious conflicts, defense mechanisms, and fantasies. Moreover, the flawed methodology of many existing studies [24] unavoidably demeans the validity of references, such as ambiguously specified patient samples and intervention practice, insufficient monitoring of treatment adherence, and absence of adequate controls. In the present and future studies, there would be potential resistance from the psychodynamic community. While psychoanalytic therapists generally emphasize patient confidentiality and independence in constructing treatment for their patients, they usually oppose conventional research methods such as recording therapy sessions, treatment regulation, and patient randomization. Among them, the most frequent issue would be the insufficiency of samples not represented in clinical practice. Finally, the psychoanalytic terms themselves would also receive oppositions, with other disciplines denying that unconscious mind, conflicts, and fantasies can be measured [89]. All the above complications would hinder the integration of different fields and long-term investigations in neuropsychodynamics.

\section{CONCLUSIONS}

Overall, this review study briefly summarizes the etiology of major depressive disorder and expands on the neuropsychodynamic model of MDD, which is based on the integrated analysis of spatiotemporal patterns spontaneous brain activity and unconscious dynamics on the psychopathological symptoms. Qualifications and limitations are elucidated in terms of possible invalidity of the cited papers, present hindrance in the psychology society, and the inherent incompatibility of the mentioned disciplines. The neuropsychodynamic model considers the association between neurology and psychodynamics and the convolutedness of subjective experience, therefore granting crucially to the biopsycho-social model of psychiatry. From the perspective of neuropsychodynamics, current psychiatry practice could benefit from the incorporation of other disciplines. It may examine how biological and psychological aspects of psychiatric diseases are associated, and better targeting of neurobiological variables to various psychodynamic terms may assist more precise psychotherapeutic treatment. Improved understanding of mental conditions such as MDD via more comprehensive pathology that embodies diverse mechanisms of the disorder (such as factors relating to genes, social environment, culture, and diet) would hopefully break down stigmas and mental illness taboos. Neuropsychodynamic practitioners should be bilingual; they should understand and speak the language of the brain and the mind. Diagnosing and treating MDD in terms of neural correlations, internal object relationships, distortions in intrapsychic structures, and epigenetics may formulate a scientific model of MDD for the future.

\section{ACKNOWLEDGMENT}

My special thanks go to Prof. Jeff Dalley, Dr. in Cambridge University, Ms. Susan Zuo in Tokyo University, Ms. Yvonne Li, and Ms. Chaya Cheng for inspiration, valuable feedback, and kind instructions.

\section{REFERENCES}

[1] World Health Organization. (2017). Depression and Other Common Mental Disorders: Global Health Estimates.

https://apps.who.int/iris/bitstream/handle/10665/25 4610/WHO-MSD-MER-2017.2-

eng.pdf;jsessionid=2A52412EC4FD7E2707B9228 C4ED61604? sequence $=1$.

[2] James, S. L. et al. Global, regional, and national incidence, prevalence, and years lived with disability for 354 diseases and injuries for 195 countries and territories, 1990-2017: A systematic analysis for the Global Burden of Disease Study 2017. Lancet, 392, 1789-1858 (2018).

[3] Hasin, D. S., Sarvet, A. L., Meyers, J. L., Saha, T. D., Ruan, W. J., Stohl, M., \& Grant, B. F. (2018). Epidemiology of Adult DSM-5 Major Depressive Disorder and Its Specifiers in the United States. JAMA Psychiatry, 75(4), 336. https://doi.org/10.1001/jamapsychiatry.2017.4602

[4] Otte, C., Gold, S. M., Penninx, B. W., Pariante, C. M., Etkin, A., Fava, M., Mohr, D. C., \& Schatzberg, A. F. (2016). Major depressive disorder. Nature Reviews Disease Primers, 2(1). https://doi.org/10.1038/nrdp.2016.65 
[5] Wray, N. R., Ripke, S., Mattheisen, M., Trzaskowski, M., Byrne, E. M., Abdellaoui, A., Adams, M. J., Agerbo, E., Air, T. M., Andlauer, T., Bacanu, S. A., Bækvad-Hansen, M., Beekman, A., Bigdeli, T. B., Binder, E. B., Blackwood, D., Bryois, J., Buttenschøn, H. N., Bybjerg-Grauholm, J., Cai, N., ... Major Depressive Disorder Working Group of the Psychiatric Genomics Consortium (2018). Genome-wide association analyses identify 44 risk variants and refine the genetic architecture of major depression. Nature genetics, 50(5), 668-681. https://doi.org/10.1038/s41588-018-0090-3

[6] Geschwind, D. H., \& Flint, J. (2015). Genetics and genomics of psychiatric disease. Science (New York, N.Y.), 349(6255), 1489-1494. https://doi.org/10.1126/science.aaa8954

[7] Hyman S. (2014). Mental health: depression needs large human-genetics studies. Nature, 515(7526), 189-191. https://doi.org/10.1038/515189a

[8] Levey, D. F., Stein, M. B., Wendt, F. R., Pathak, G. A., Zhou, H., Aslan, M., Quaden, R., Harrington, K. M., Nuñez, Y. Z., Overstreet, C., Radhakrishnan, K., Sanacora, G., McIntosh, A. M., Shi, J., Shringarpure, S. S., Concato, J., Polimanti, R., \& Gelernter, J. (2021). Bi-ancestral depression GWAS in the Million Veteran Program and metaanalysis in $>1.2$ million individuals highlight new therapeutic directions. Nature Neuroscience, 24(7), 954-963. https://doi.org/10.1038/s41593-02100860-2

[9] Kendler, K. S., \& Gardner, C. O. (2016). Depressive vulnerability, stressful life events and episode onset of major depression: a longitudinal model. Psychological medicine, 46(9), 1865-1874. https://doi.org/10.1017/S0033291716000349

[10] Lasič, E., Lisjak, M., Horvat, A., Božić, M., Šakanović, A., Anderluh, G., Verkhratsky, A., Vardjan, N., Jorgačevski, J., Stenovec, M., \& Zorec, R. (2019). Astrocyte Specific Remodeling of Plasmalemmal Cholesterol Composition by Ketamine Indicates a New Mechanism of Antidepressant Action. Scientific Reports, 9(1). https://doi.org/10.1038/s41598-019-47459-z

[11] Borsini, A., Nicolaou, A., Camacho-Muñoz, D., Kendall, A. C., Di Benedetto, M. G., Giacobbe, J., Su, K.-P., \& Pariante, C. M. (2021). Omega-3 polyunsaturated fatty acids protect against inflammation through production of LOX and CYP450 lipid mediators: relevance for major depression and for human hippocampal neurogenesis. Molecular Psychiatry. https://doi.org/10.1038/s41380-021-01160-8
[12] Kim, H.-D., Wei, J., Call, T., Quintus, N. T., Summers, A. J., Carotenuto, S., Johnson, R., Ma, X., Xu, C., Park, J. G., Qiu, S., \& Ferguson, D. (2021). Shisa6 mediates cell-type specific regulation of depression in the nucleus accumbens. Molecular Psychiatry. https://doi.org/10.1038/s41380-02101217-8

[13] Taliaz, D., Spinrad, A., Barzilay, R., Barnett-Itzhaki, Z., Averbuch, D., Teltsh, O., Schurr, R., DarkiMorag, S., \& Lerer, B. (2021). Optimizing prediction of response to antidepressant medications using machine learning and integrated genetic, clinical, and demographic data. Translational Psychiatry, 11(1). https://doi.org/10.1038/s41398-021-01488-3

[14] Ayano, G., Demelash, S., Yohannes, Z., Haile, K., Tulu, M., Assefa, D., Tesfaye, A., Haile, K., Solomon, M., Chaka, A., \& Tsegay, L. (2021). Misdiagnosis, detection rate, and associated factors of severe psychiatric disorders in specialized psychiatry centers in Ethiopia. Annals of general psychiatry, 20(1), 10. https://doi.org/10.1186/s12991-021-00333-7

[15] Puyat, J. H., Kazanjian, A., Goldner, E. M., \& Wong, H. (2016). How Often Do Individuals with Major Depression Receive Minimally Adequate Treatment? A Population-Based, Data Linkage Study. Canadian Journal of Psychiatry. Revue Canadienne de Psychiatrie, 61(7), 394-404. https://doi.org/10.1177/0706743716640288

[16] Gold, S. M., Köhler-Forsberg, O., \& Moss-Morris, R. (2020). Comorbid depression in medical diseases. Nature Reviews Disease Primers, 6(69). https://doi.org/https://doi.org/10.1038/s41572-0200200-2

[17] Cipriani, A., Furukawa, T. A., Salanti, G., Geddes, J. R., Higgins, J. P., Churchill, R., Watanabe, N., Nakagawa, A., Omori, I. M., McGuire, H., Tansella, M., \& Barbui, C. (2009). Comparative efficacy and acceptability of 12 new-generation antidepressants: a multiple-treatments meta-analysis. Lancet (London, England), 373(9665), 746-758. https://doi.org/10.1016/S0140-6736(09)60046-5

[18] Zhou, X., Cipriani, A., Furukawa, T. A., Cuijpers, P., Zhang, Y., Hetrick, S. E., Pu, J., Yuan, S., Del Giovane, C., \& Xie, P. (2018). Comparative efficacy and tolerability of new-generation antidepressants for major depressive disorder in children and adolescents: protocol of an individual patient data meta-analysis. BMJ Open, 8(1). https://doi.org/10.1136/bmjopen-2017-018357 
[19] Conradi, H. J., Ormel, J., \& de Jonge, P. (2011). Presence of individual (residual) symptoms during depressive episodes and periods of remission: a 3year prospective study. Psychological medicine, 41(6),

$1165-1174$ https://doi.org/10.1017/S0033291710001911

[20] Borsboom D, Cramer AOJ, Kalis A. (2019) Brain disorders? Not really: Why network structures block reductionism in psychopathology research. Behavioral and Brain Sciences 42, e2: 1-63. doi:10.1017/ S0140525X17002266

[21] Goldberg D. (2011). The heterogeneity of "major depression". World psychiatry : official journal of the World Psychiatric Association (WPA), 10(3), 226-228. https://doi.org/10.1002/j.20515545.2011.tb00061.x

[22] Dennett, D. C. (1987). The intentional stance. The MIT Press.

[23] Boeker, H. (2020). Psychodynamic psychiatry and neurobiology. Swiss Archives of Neurology, Psychiatry and Psychotherapy. https://doi.org/10.4414/sanp.2020.03140

[24] Yakeley, J. (2018). Psychoanalysis in modern mental health practice. The Lancet Psychiatry, 5(5), 443-450. https://doi.org/10.1016/s22150366(18)30052-X

[25] Midgley, N., Mortimer, R., Cirasola, A., Batra, P., \& Kennedy, E. (2021). The Evidence-Base for Psychodynamic Psychotherapy With Children and Adolescents: A Narrative Synthesis. Frontiers in psychology, 12 , 662671 https://doi.org/10.3389/fpsyg.2021.662671

[26] Jennissen, S., Connolly Gibbons, M. B., CritsChristoph, P., Schauenburg, H., \& Dinger, U. (2021). Insight as a mechanism of change in dynamic therapy for major depressive disorder. Journal of Counseling Psychology. Advance online publication. https://doi.org/10.1037/cou0000554

[27] Ajilchi, B., Nejati, V., Town, J. M., Wilson, R., \& Abbass, A. (2016). Effects of Intensive Short-Term Dynamic Psychotherapy on Depressive Symptoms and Executive Functioning in Major Depression. Journal of Nervous \& Mental Disease, 204(7), 500 505 .

https://doi.org/10.1097/nmd.0000000000000518

[28] Leichsenring, F., \& Rabung, S. (2008). Effectiveness of long-term psychodynamic psychotherapy: a meta-analysis. JAMA, 300(13), $1551-1565$

https://doi.org/10.1001/jama.300.13.1551
[29] Gabbard, G. O. (2010). Long-term psychodynamic psychotherapy: A basic text. American Psychiatric Publishing, Inc..

[30] Ribeiro, Â., Ribeiro, J. P., \& von Doellinger, O. (2017). Depression and psychodynamic psychotherapy. Revista Brasileira De Psiquiatria, 40(1), 105-109. https://doi.org/10.1590/15164446-2016-2107

[31] Gabbard, G. (2014). Psychodynamic Psychiatry in Clinical Practice. (5th ed.). American Psychiatric Publishing.

[32] Salone, A., Di Giacinto, A., Lai, C., De Berardis, D., Iasevoli, F., Fornaro, M., De Risio, L., Santacroce, R., Martinotti, G., \& Giannantonio, M. D. (2016). The Interface between Neuroscience and NeuroPsychoanalysis: Focus on Brain Connectivity. Frontiers in human neuroscience, 10, 20. https://doi.org/10.3389/fnhum.2016.00020

[33] Boeker, H., Hartwich, P., \& Northoff, G. (2018). Neuropsychodynamic Psychiatry. Springer International Publishing.

[34] Solms, M., \& Panksepp, J. (2012). The "Id" Knows More than the "Ego" Admits: Neuropsychoanalytic and Primal Consciousness Perspectives on the Interface Between Affective and Cognitive Neuroscience. Brain Sciences, 2(2), 147-175. https://doi.org/10.3390/brainsci2020147

[35] Bakken, T. E., Miller, J. A., Ding, S. L., Sunkin, S. M., Smith, K. A., Ng, L., etc. (2016). A comprehensive transcriptional map of primate brain development. Nature, 535(7612), 367-375. https://doi.org/10.1038/nature18637

[36] Fujii, T., Sakurai, A., Littleton, J. T., \& Yoshihara, M. (2021). Synaptotagmin 7 switches short-term synaptic plasticity from depression to facilitation by suppressing synaptic transmission. Scientific Reports, 11(1). https://doi.org/10.1038/s41598021-83397-5

[37] Pervanidou, P., \& Chrousos, G. P. (2012). Posttraumatic Stress Disorder in Children and Adolescents: Neuroendocrine Perspectives. Science Signaling, 5(245). https://doi.org/10.1126/scisignal.2003327

[38] Alhassen, S., Chen, S., Alhassen, L., Phan, A., Khoudari, M., De Silva, A., Barhoosh, H., Wang, Z., Parrocha, C., Shapiro, E., Henrich, C., Wang, Z., Mutesa, L., Baldi, P., Abbott, G. W., \& Alachkar, A. (2021). Intergenerational trauma transmission is associated with brain metabotranscriptome remodeling and mitochondrial dysfunction. 
Communications Biology, 4(1). https://doi.org/10.1038/s42003-021-02255-2

[39] Jami, E. S., Hammerschlag, A. R., Bartels, M., \& Middeldorp, C. M. (2021). Parental characteristics and offspring mental health and related outcomes: a systematic review of genetically informative literature. Translational Psychiatry, 11(1). https://doi.org/10.1038/s41398-021-01300-2

[40] Newland, R. P., \& Crnic, K. A. (2017). Developmental Risk and Goodness of Fit in the Mother-Child Relationship: Links to Parenting Stress and Children's Behaviour Problems. Infant and child development, 26(2), e1980. https://doi.org/10.1002/icd.1980

[41] Fox, A. S., Harris, R. A., Rosso, L. D., Raveendran, M., Kamboj, S., Kinnally, E. L., Capitanio, J. P., \& Rogers, J. (2021). Infant inhibited temperament in primates predicts adult behavior, is heritable, and is associated with anxiety-relevant genetic variation. Molecular Psychiatry. https://doi.org/10.1038/s41380-021-01156-4

[42] Feldman, R., Braun, K., \& Champagne, F. A. (2019). The neural mechanisms and consequences of paternal caregiving. Nature Reviews Neuroscience, 20(4), 205-224. https://doi.org/10.1038/s41583019-0124-6

[43] Freud, S. (1938). An Outline of Psychoanalysis, Standard Edn. Vol. 23. London: Hogarth Press, 144-205.

[44] Solms, M. (2018). "The unconscious" in psychoanalysis and neuroscience: an integrated approach to the cognitive unconscious*. Unrepressed Unconscious, Implicit Memory, and Clinical Work, 1-25. https://doi.org/10.4324/9780429484629-1

[45] Sohtorik İ lkmen, Y., \& Halfon, S. (2019). Transference interpretations as predictors of increased insight and affect expression in a single case of long-term psychoanalysis. Research in psychotherapy (Milano), 22(3), 408. https://doi.org/10.4081/ripppo.2019.408

[46] Freud, S. (1917). Mourning and Melancholia. The Standard Edition of the Complete Psychological Works of Sigmund Freud, Volume XIV (1914 1916): On the History of the Psycho-Analytic Movement, Papers on Metapsychology and Other Works, 237-258

[47] Boeker, H., \& Northoff, G. (2018). Depressive Syndromes. Neuropsychodynamic Psychiatry, 219-272. https://doi.org/10.1007/978-3-319$75112-2 \quad 11$
[48] Boeker, H., \& Kraehenmann, R. (2018). Neuropsychodynamic Approach to Depression: Integrating Resting State Dysfunctions of the Brain and Disturbed Self-Related Processes. Frontiers in Human Neuroscience, 12. https://doi.org/10.3389/fnhum.2018.00247

[49] Carhart-Harris R. L., Mayberg H. S., Malizia A. L., Nutt D. (2008). Mourning and melancholia revisited: correspondences between principles of Freudian metapsychology and empirical findings in neuropsychiatry. Ann. Gen. Psychiatry 24, 7-9. 10.1186/1744-859X-7-9

[50] Bowlby J. (1980). Attachment and Loss: Sadness and Depression (Vol. 3). New York, NY: Basic Books.

[51] Yamamura, T., Okamoto, Y., Okada, G., Takaishi, Y., Takamura, M., Mantani, A., Kurata, A., Otagaki, Y., Yamashita, H., \& Yamawaki, S. (2016). Association of thalamic hyperactivity with treatment-resistant depression and poor response in early treatment for major depression: a resting-state fMRI study using fractional amplitude of lowfrequency fluctuations. Translational Psychiatry, 6(3). https://doi.org/10.1038/tp.2016.18

[52] Peng, X., Lau, W. K., Wang, C., Ning, L., \& Zhang, R. (2020). Impaired left amygdala resting state functional connectivity in subthreshold depression individuals. Scientific Reports, 10(1). https://doi.org/10.1038/s41598-020-74166-x

[53] Yoshino, A., Okamoto, Y., Doi, M., Otsuru, N., Okada, G., Takamura, M., Ichikawa, N., Yokoyama, S., Yamashita, H., \& Yamawaki, S. (2017). Regional brain functions in the resting state indicative of potential differences between depression and chronic pain. Scientific Reports, 7(1). https://doi.org/10.1038/s41598-017-03522-1

[54] Phillips, M. L., Drevets, W. C., Rauch, S. L., \& Lane, R. (2003). Neurobiology of emotion perception II: Implications for major psychiatric disorders. Biological psychiatry, 54(5), 515-528. https://doi.org/10.1016/s0006-3223(03)00171-9

[55] Alcaro, A., Panksepp, J., Witczak, J., Hayes, D. J., \& Northoff, G. (2010). Is subcortical-cortical midline activity in depression mediated by glutamate and GABA? A cross-species translational approach. Neuroscience and biobehavioral reviews, 34(4), 592-605. https://doi.org/10.1016/j.neubiorev.2009.11.023

[56] Drysdale, A. T., Grosenick, L., Downar, J., Dunlop, K., Mansouri, F., Meng, Y., etc. (2016). Restingstate connectivity biomarkers define neurophysiological subtypes of depression. Nature 
Medicine,

23(1),

$28-38$.

https://doi.org/10.1038/nm.4246

[57] Bonk, S., Hertel, J., Zacharias, H. U., Terock, J., Janowitz, D., Homuth, G., Nauck, M., Völzke, H., Meyer zu Schwabedissen, H., Van der Auwera, S., \& Grabe, H. J. (2020). Vitamin D moderates the interaction between 5-HTTLPR and childhood abuse in depressive disorders. Scientific Reports, 10(1). https://doi.org/10.1038/s41598-020-79388-7

[58] Clewell, T. (2004). Mourning Beyond Melancholia: Freud's Psychoanalysis of Loss. Journal of the American Psychoanalytic Association, 52(1), 4367.

https://doi.org/10.1177/00030651040520010601

[59] Qin P., Northoff G. (2011). How is our self related to midline regions and the default-mode network? Neuroimage, 57, 1221-1233. 10.1016/j.neuroimage.2011.05.028

[60] Chase, H. W., Auerbach, R. P., Brent, D. A., Posner, J., Weissman, M. M., \& Talati, A. (2021). Dissociating default mode network resting state markers of suicide from familial risk factors for depression. Neuropsychopharmacology. https://doi.org/10.1038/s41386-021-01022-5

[61] Kaiser, R. H., Kang, M. S., Lew, Y., Van Der Feen, J., Aguirre, B., Clegg, R., Goer, F., Esposito, E., Auerbach, R. P., Hutchison, R. M., \& Pizzagalli, D. A. (2019). Abnormal frontoinsular-default network dynamics in adolescent depression and rumination: a preliminary resting-state co-activation pattern analysis. Neuropsychopharmacology, 44(9), 1604 1612. https://doi.org/10.1038/s41386-019-0399-3

[62] Zhu, X., Zhu, Q., Shen, H., Liao, W., \& Yuan, F. (2017). Rumination and Default Mode Network Subsystems Connectivity in First-episode, DrugNaive Young Patients with Major Depressive Disorder. Scientific Reports, 7(1). https://doi.org/10.1038/srep43105

[63] Deci, E. L., Eghrari, H., Patrick, B. C., \& Leone, D. R. (1994). Facilitating Internalization: The SelfDetermination Theory Perspective. Journal of Personality, 62(1), 119-142. doi:10.1111/j.14676494.1994.tb00797.x

[64] Blatt, S. J. (1998). Contributions of Psychoanalysis To the Understanding and Treatment of Depression. Journal of the American Psychoanalytic Association, 46(3), 723-752. https://doi.org/10.1177/00030651980460030301

[65] LaFarge, L. (2012). Defense and resistance. In G. O. Gabbard, B. E. Litowitz, \& P. Williams
(Eds.), Textbook of psychoanalysis (pp. 93-104). American Psychiatric Publishing, Inc.

[66] Cramer, P. (2015). Understanding Defense Mechanisms. Psychodynamic Psychiatry, 43(4), $523-552$.

https://doi.org/10.1521/pdps.2015.43.4.523

[67] Brenner, C. (1991). A Psychoanalytic Perspective on Depression. Journal of the American Psychoanalytic Association, 39(1), 25-43. https://doi.org/10.1177/000306519103900102

[68] Rosenbaum, D., Haipt, A., Fuhr, K., Haeussinger, F. B., Metzger, F. G., Nuerk, H.-C., Fallgatter, A. J., Batra, A., \& Ehlis, A.-C. (2017). Aberrant functional connectivity in depression as an index of state and trait rumination. Scientific Reports, 7(1). https://doi.org/10.1038/s41598-017-02277-z

[69] Satterthwaite, T. D., Kable, J. W., Vandekar, L., Katchmar, N., Bassett, D. S., Baldassano, C. F., Ruparel, K., Elliott, M. A., Sheline, Y. I., Gur, R. C., Gur, R. E., Davatzikos, C., Leibenluft, E., Thase, M. E., \& Wolf, D. H. (2015). Common and Dissociable Dysfunction of the Reward System in Bipolar and Unipolar Depression. Neuropsychopharmacology, 40(9), 2258-2268. https://doi.org/10.1038/npp.2015.75

[70] Rupprechter, S., Stankevicius, A., Huys, Q. J., Steele, J. D., \& d, P. (2018). Major Depression Impairs the Use of Reward Values for DecisionMaking. Scientific Reports, 8(1). https://doi.org/10.1038/s41598-018-31730-w

[71] Lydon-Staley, D. M., Kuehner, C., Zamoscik, V., Huffziger, S., Kirsch, P., \& Bassett, D. S. (2019). Repetitive negative thinking in daily life and functional connectivity among default mode, fronto-parietal, and salience networks. Translational Psychiatry, 9(1). https://doi.org/10.1038/s41398-019-0560-0

[72] Parker, D. B., \& Razlighi, Q. R. (2019). Taskevoked Negative BOLD Response and Functional Connectivity in the Default Mode Network are Representative of Two Overlapping but Separate Neurophysiological Processes. Scientific Reports, 9(1). https://doi.org/10.1038/s41598-019-50483-8

[73] Smoski M. J., Felder J., Bizzell J., Green S. R., Ernst M., Lynch T. R., et al. . (2009). fMRI of alterations in reward selection, anticipation and feedback in major depressive disorder. J. Affect. Disord. 118, 69-78. 10.1016/j.jad.2009.01.034

[74] Grimm S., Schmidt C. F., Bermpohl F., Heinzel A., Dahlem Y., Wyss M., et al. . (2006). Segregated neural representation of distinct emotion 
dimensions in the prefrontal cortex-an fMRI study. Neuroimage 30, $325-340$ 10.1016/j.neuroimage.2005.09.006

[75] Wiebking C., Bauer A., de Greck M., Duncan N. W., Tempelmann C., Northoff G. (2010). Abnormal body perception and neural activity in the insula in depression: an fMRI study of the depressed "material me". World J. Biol. Psychiatry, 11, 538549.

[76] Karabatsiakis, A., \& Schönfeldt-Lecuona, C. (2020). Depression, mitochondrial bioenergetics, and electroconvulsive therapy: a new approach towards personalized medicine in psychiatric treatment - a short review and current perspective. Translational Psychiatry, 10(1). https://doi.org/10.1038/s41398020-00901-7

[77] Northoff, G., \& Sibille, E. (2014). Cortical GABA neurons and self-focus in depression: a model linking cellular, biochemical and neural network findings. Molecular Psychiatry, 19(9), 959-959. https://doi.org/10.1038/mp.2014.108

[78] Wagner, G., Sinsel, E., Sobanski, T., Köhler, S., Marinou, V., Mentzel, H. J., Sauer, H., \& Schlösser, R. G. (2006). Cortical inefficiency in patients with unipolar depression: an event-related FMRI study with the Stroop task. Biological psychiatry, 59(10), 958-965.

https://doi.org/10.1016/j.biopsych.2005.10.025

[79] Baars, B. J., \& Gage, N. M. (2018). Fundamentals of cognitive neuroscience: a beginner's guide. Elsevier/Academic Press.

[80] Ho, T. C., Sacchet, M. D., Connolly, C. G., Margulies, D. S., Tymofiyeva, O., Paulus, M. P., Simmons, A. N., Gotlib, I. H., \& Yang, T. T. (2017). Inflexible Functional Connectivity of the Dorsal Anterior Cingulate Cortex in Adolescent Major Depressive Disorder. Neuropsychopharmacology, 42(12), 2434-2445. https://doi.org/10.1038/npp.2017.103

[81] Le-Niculescu, H., Roseberry, K., Gill, S. S., Levey, D. F., Phalen, P. L., Mullen, J., Williams, A., Bhairo, S., Voegtline, T., Davis, H., Shekhar, A., Kurian, S. M., \& Niculescu, A. B. (2021). Precision medicine for mood disorders: objective assessment, risk prediction, pharmacogenomics, and repurposed drugs. Molecular Psychiatry. https://doi.org/10.1038/s41380-021-01061-w

[82] De Roten, Y., Djillali, S., Crettaz von Roten, F., Despland, J.-N., \& Ambresin, G. (2021). Defense Mechanisms and Treatment Response in Depressed Inpatients. Frontiers in Psychology, 12. https://doi.org/10.3389/fpsyg.2021.633939
[83] Cuijpers, P., Sijbrandij, M., Koole, S. L., Andersson, G., Beekman, A. T., \& Reynolds, C. F., 3rd (2014). Adding psychotherapy to antidepressant medication in depression and anxiety disorders: a meta-analysis. World psychiatry : official journal of the World Psychiatric Association (WPA), 13(1), 56-67. https://doi.org/10.1002/wps.20089

[84] Zarbo, C., Tasca, G. A., Cattafi, F., \& Compare, A. (2016). Integrative Psychotherapy Works. Frontiers in psychology, 6, 2021. https://doi.org/10.3389/fpsyg.2015.02021

[85] Goodwin N. (2016). Understanding Integrated Care. International journal of integrated care, 16(4), 6. https://doi.org/10.5334/ijic.2530

[86] Northoff G. (2018) Self Between Brain and World: Neuropsychodynamic Approach, Social Embedded Brain and Relational Self. In: Boeker H., Hartwich P., Northoff G. (eds) Neuropsychodynamic Psychiatry. Springer, Cham. https://doi.org/10.1007/978-3-319-75112-2_4

[87] Metzinger, T. (2003). Being no one: The self-model theory of subjectivity. MIT Press.

[88] Northoff, G., Bermpohl, F., Schoeneich, F., \& Boeker, H. (2007). How does our BRAIN CONSTITUTE DEFENSE Mechanisms? Firstperson neuroscience and psychoanalysis. Psychotherapy and Psychosomatics, 76(3), 141153. https://doi.org/10.1159/000099841

[89] Yakeley, J. (2014). Psychodynamic psychotherapy: Developing the evidence base. Advances in Psychiatric Treatment, 20(4), 269-279. doi:10.1192/apt.bp.113.012054 